\title{
Screening of nasal carriage for Staphylococcus aureus and their resistance to oxacillin and cefoxitin among medical students in Karbala University
}

\author{
Suhad Hadi Mohammed, Mohammed Neama Hmood, Areej Abbas Abd, Seham Aaid Obaid, \\ Baraa Ahmed Fahad \& Fatemah Hadi Kadhem
}

\begin{abstract}
Objective Carriage of Staphylococcus aureus, especially methicillin-resistant S. aureus (MRSA) is a problem within healthcare organizations and in the community. The aim of the study was to screen S. aureus carriage and their susceptibility to cefoxitin and oxacillin among medical students.

Methods A total of 100 nasal swabs were collected. Isolation and identification of the isolate as S. aureus was done using Gram stain, coagulase test and catalase test. S. aureus isolates were confirmed as MRSA using cefoxitin ( $30 \mu \mathrm{g})$ disc and oxacillin ( $30 \mu \mathrm{g})$ disc by KirbyBauer disc diffusion method on Mueller-Hinton agar. From 100 nasal swabs, 76 were coagulase negative Staphylococci and 20 were coagulase positive Staphylococci.

Results From 100 nasal swabs, 76 were coagulase negative Staphylococci and 20 were coagulase positive Staphylococci. From these, 60\% and $40 \%$ were oxacillin- and cefoxitin-resistant isolates, respectively. The data obtained from this study revealed that there were carriers of MRSA among the medical students.

Keywords MRSA, coagulase, oxacillin, cefoxitin
\end{abstract}

\section{Introduction}

Staphylococcus aureus has long been recognized as an important human pathogen. ${ }^{1}$ The anterior nares represent the primary ecological reservoir of $S$. aureus in humans, and nasal carriage is a major risk factor for a variety of infections. ${ }^{2}$ Three patterns of nasal carriages are known (persistent carrier, intermittent, and non-carrier). Approximately $20 \%$ of the individuals almost always carry one type of strain and they are called persistent carriers. A large proportion of the population (60\%) harbours S. aureus intermittently, and the strains change with varying frequencies. Such persons are called intermittent carriers. Finally, a minority of the people (20\%) almost never carry S. aureus and they are called noncarriers. A persistent carriage is more common in children than in adults, and many people change their pattern of carriage between the age of 10 and 20 years. The reasons for these differences in the colonization patterns are unknown. Healthcare workers (HCWs) constitute an important reservoir of $S$. aureus. Several studies have reported that the rate of the nasal carriage of $S$. aureus among the HCWs ranges from $16.8 \%$ to $56.1 \% .^{4-7}$

Treatment of infection caused by $S$. aureus has become more problematic since the development of antimicrobialresistant $S$. aureus (MRSA). As the MRSA strains are resistant to all $\beta$-lactam antibiotics, the treatment options are limited significantly. The incidence of nosocomial infection caused by MRSA continues to increase worldwide. Infections caused by MRSA strains are associated with longer hospital stay, prolonged antibiotic administration and higher costs than infections caused by methicillin-susceptible S. aureus (MSSA) strains.

By definition, MRSA strains harbours the mecA gene, which encodes the low-affinity penicillin-binding protein (PBP) designated as PBP2a. ${ }^{9,10}$
The Clinical and Laboratory Standards Institute (CLSI) states that the oxacillin and cefoxitin disk tests are equivalent in sensitivity and specificity for the detection of mecAmediated resistance in $S$. aureus. ${ }^{11}$

The screening of the nasal carriage in HCWs is an important component in the control of MRSA in any healthcare facility. The identification of the colonized staff members allows an appropriate management of these persons to prevent the spread of organism within hospitals or in communities. Because medical students belong to the $\mathrm{HCW}$ in future, the aim of our study is to screen $S$. aureus nasal carriage of these individuals, and to identify the prevalence of oxacillin and cefoxitin resistance among the isolated S. aureus.

\section{Materials and Methods}

\section{Specimen Collections}

Anterior nasal swabs were taken from 100 healthy students from third and fourth stage whom they had internship programme in hospitals during summer from October 2014 to March 2015. Sterile swab was moistened with sterile normal saline and was rotated at least 5 times in both nares, then placed in the transport media, using standard methods. ${ }^{12-14}$

\section{Specimens Processing}

All specimens were directly inoculated from transport media into plates of Mannitol salt agar (MSA) and blood agar and incubated at $37^{\circ} \mathrm{C}$ for $24 \mathrm{~h}$ (Fig. 1). All colonies from primary cultures were subcultured onto MSA and incubated at $37^{\circ} \mathrm{C}$ for $24 \mathrm{~h} .{ }^{15} \mathrm{~S}$. aureus were identified depending on the morphological features on culture media (beta-hemolytic on blood agar and mannitol fermentation on MSA) and biochemical tests (catalase positive test and coagulase positive 
test) also gram staining showed grampositive grape-like clusters (Fig. 1).

\section{Antibiotic Susceptibility Test}

All S. aureus isolates were tested for cefoxitin and oxacillin susceptibility by Kirby-Bauer method on Mueller-Hinton agar (MHA) (Hi-media). Plates were incubated at $37^{\circ} \mathrm{C}$ for $24 \mathrm{~h}$. Following the incubation, the inhibition zone diameter was measured. Identification of MRSAs were done by following CLSI. ${ }^{16}$ Isolates were considered susceptible to oxacillin and cefoxitin if the zone of inhibition around the disks was $\geq 22 \mathrm{~mm}$, and resistant if the zone was $\leq 21 \mathrm{~mm}$ (Fig. 2).
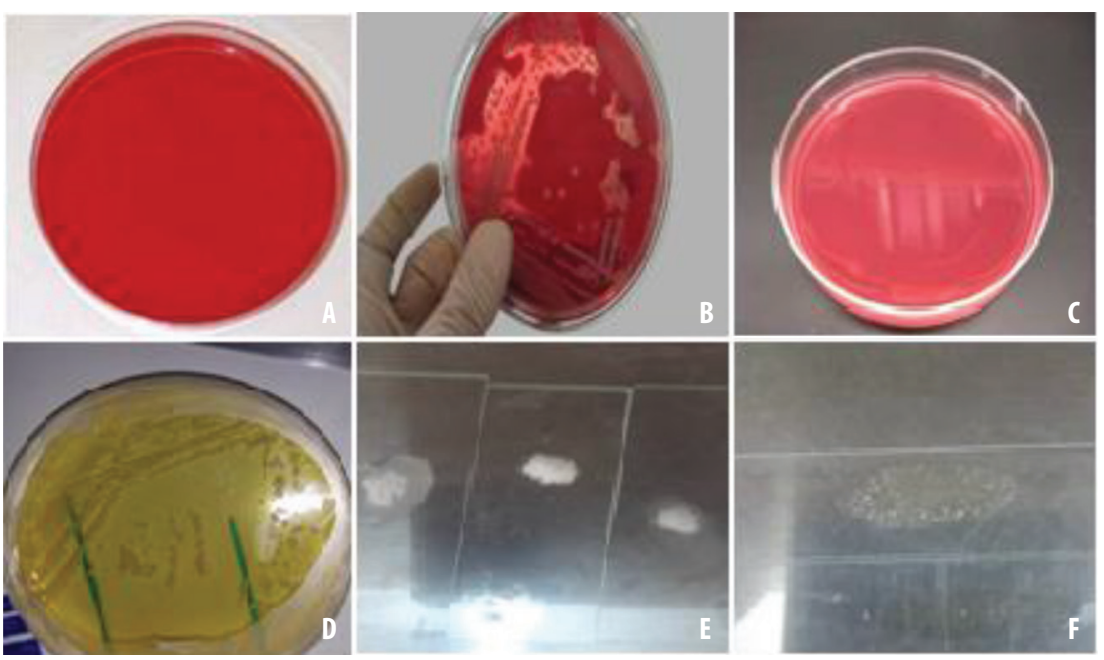

Fig. 1 A: Blood agar plate; B: beta hemolytic isolates; C: Mannitol salt agar; D: Mannitol salt agar plate with S. aureus isolates; E: catalase test; F: coagulase test.
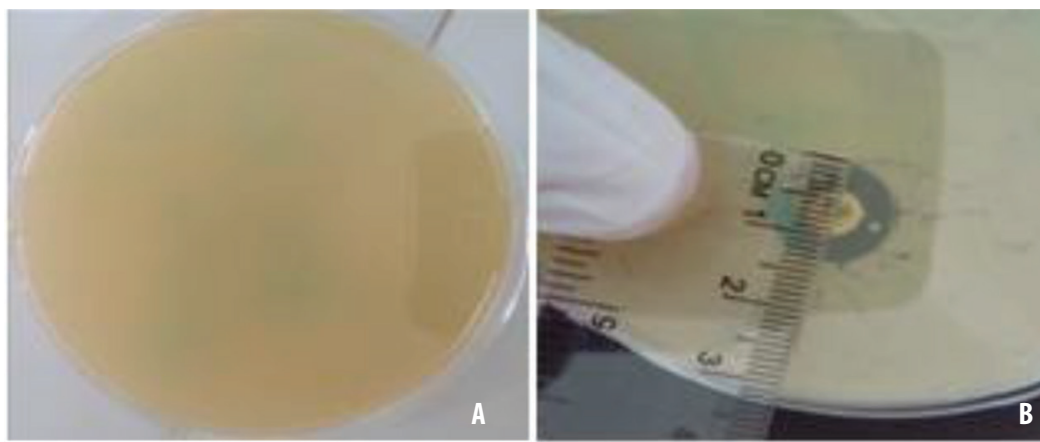

Fig. 2 A: Mueller-Hinton agar without antibiotic disc with bacterial colonies (control); B: Mueller-Hinton agar with antibiotic disc. were resistant to cefoxitin and $12(60 \%)$ were resistant to oxacillin. There is positive significant correlation between the two drugs (Table 2).

\section{Distribution of the Resistant Strains Among Sex and Age}

The prevalence of $S$. aureus nasal carriage was higher among the older age group individuals $(70 \%)$ than the younger age group (30\%). Concerning the resistance profile, there were $62.5 \%$ $(5 / 8)$ of cefoxitin-resistant isolates, and $66.6 \%(8 / 12)$ of oxacillin-resistant isolates found among the older than the younger volunteers (Table 3 ). The current study revealed that $62.5 \%(5 / 8)$ of cefoxitin-resistant isolates and 50\% $(6 / 12)$ of oxacillin-resistant isolates were found in females.

\section{Discussion}

The primary reservoir of $S$. aureus in humans is the anterior nares. Nasal carriage is recognized as a major risk factor for the development of both community-acquired and nosocomial infections. ${ }^{17,18}$ This appears to play a key role in the epidemiology and pathogenesis of infection. ${ }^{17,18}$ The factors that distinguish between a carrier and a non-carrier are still unknown. Enhanced adhesion of $S$. aureus, to cell associated and cell-free secretions, along with the induction of reduced mucociliary activity, could well explain the nasal colonization by $S$. aureus. It is imperative that nasal carriage due to $S$. aureus strains should be prevented in order to stem the rate of infection, and in preventing the transmission of resistant strains of the organism. ${ }^{8}$

Although nasal carriage of $S$. aureus is harmless in healthy individuals, they can become carriers who could pose the risk of spreading infections to the community at large, and since the section of individuals under this study were medical students, their interaction and exposure to hospital environment could cause major risks in transmitting to patients

\section{Table 1. Culture results of nasal swabs}

\begin{tabular}{lcc}
\hline Culture results & Isolates & N (\%) \\
\hline \multirow{2}{*}{ Culture positive } & S. aureus & $20(\%)$ \\
& Coagulase negative Staphylococci & $76(\%)$ \\
Culture positive & Micrococcus & 3 \\
Other bacteria & Bacillus & 1 \\
\hline
\end{tabular}

Table 2. Screening for MRSA

\begin{tabular}{lccc}
\hline Antibiotic & Resistant N (\%) & Sensitive N (\%) & Total N (\%) \\
\hline Oxacillin & $12(60)$ & $8(40)$ & $20(100)$ \\
Cefoxitin & $8(40)$ & $12(60)$ & $20(100)$ \\
Correlation & $r=0.667$ P value $=0.001^{*}$ & \\
\hline
\end{tabular}

MRSA: methicillin-resistant S. aureus. *Correlation significant at 0.01 level. 
Table 3. Distribution of S. aureus isolates among age group

\begin{tabular}{|c|c|c|c|c|c|c|}
\hline & & S. aureus N (\%) & 0xacillin & ensitivity & Cefoxitin & ensitivity \\
\hline & & & Sensitive & Resistance & Sensitive & Resistance \\
\hline A rar & Younger & $6(30)$ & 2 & 4 & 3 & 3 \\
\hline Age groups & Older & $14(70)$ & 6 & 8 & 9 & 5 \\
\hline Gov & Male & $10(50)$ & 4 & 6 & 7 & 3 \\
\hline sex & Female & $10(50)$ & 4 & 6 & 5 & 5 \\
\hline Total & & $20(100)$ & 8 & 12 & 12 & 8 \\
\hline
\end{tabular}

and spreading nosocomial infections. Therefore, it is necessary to detect $S$. aureus carriage in medical students.

The current study revealed that out of the 100 samples collected from medical students, 96 were identified as Staphylococcus while 4 specimens showed no growth of Staphylococcal colonies, instead they showed growth of Micrococcus sp. and Bacillus. Twenty samples out of 96 (20.8\%) were coagulase positive $S$. aureus isolates and $76(79.1 \%)$ were coagulase negative Staphylococci.

The prevalence of $S$. aureus carriage has been reported in healthy populations in several countries; $43.2 \%$ of $S$. aureus in nasal cavity of adults in Iraq, $17.3 \%$ in nasal cavity of Turkish children, 36\% in nares of Japanese adults and 32.4\% in nasal cavity of adults in the United States. ${ }^{19-22}$ Pant and Rai's (2007) ${ }^{23}$ findings revealed higher $S$. aureus nasal colonization rate $(43.8 \%)$ in staffs of teaching hospital in Nepal. Also, in Abia state of Nigeria, Chigbu and Ezeronye $(2003)^{24}$ reported 50\% nasal colonization in both hospital and nonhospital subjects. Chatterjee et al. (2009) ${ }^{25}$ showed that the overall prevalence of $S$. aureus nasal colonization was $52.3 \%$. Whereas Onanuga and Temedie $(2011)^{26}$ showed that $33.3 \%$ S. aureus isolates were obtained from 120 nares specimens screened. Whilst, Adesida et al. (2007) ${ }^{27}$ reported a much lower $(14.0 \%)$ nasal colonization in medical students in Lagos, Nigeria. These variations may be attributed to the characteristics of the population under study. A population that is on antibiotics at the time of sampling may yield a much lower prevalence of $S$. aureus while a population from hospital settings may yield a much higher prevalence because of the high prevalence of infectious patients in that environment. Other factors that can cause variations may be sampling and culture techniques.
Concerning the detection of MRSA, all the $20 \mathrm{~S}$. aureus isolates were tested for cefoxitin and oxacillin resistance using a disk diffusion method and $60 \%$ and $40 \%$ were found to be susceptible to oxacillin and cefoxitin, respectively. Higher incidence of MRSA was found in another study in Iraq/Baghdad among HCWs and patients in hospital. ${ }^{28}$ In another study, resistant percentage was found to be of $90.9 \% .^{29}$ Fey et al. $(2003)^{30}$ stated that the resistance to methicillin was $81 \%$, while Jain et al. $(2008)^{31}$ observed about $75.26 \%$ of isolates were methicillin-resistant. These observed differences may due to the variation in the geographic area, sources of clinical specimens, genetic background and the collection site of the isolates. ${ }^{31}$

\section{Conclusion}

The data obtained from this study revealed that there were reservoirs or carriers of MRSA in medical students.

Screening for resistant strains of Staphylococci in medical students should be adopted as a protocol in medical colleges, in order to curb the spread of drug-resistant Staphylococci from the hospital to the community. This will also help in monitoring the HCWs population who might pose a risk to patients and hospital personnel and the community, at large.

\section{References}

1. Rongpharpi SR, Hazarika NK, Kalita H. The prevalence of nasal carriage of Staphylococcus aureus among healthcare workers at a tertiary care hospital in Assam with special reference to MRSA. J Clin Diagn Res. 2013 Feb;7(2):257-60 doi: http://dx.doi.org/10.7860/JCDR/2013/4320.2741 PMID: 23543837

2. van den Akker EL, Nouwen JL, Melles DC, van Rossum EF, Koper JW, Uitterlinden AG, et al. Staphylococcus aureus nasal carriage is associated with glucocorticoid receptor gene polymorphisms. J Infect Dis. 2006 Sept 15; 194(6):814-8. doi: http://dx.doi.org/10.1086/506367 PMID: 16941349

3. Kluytmans J, van Belkum A, Verbrugh H. Nasal carriage of Staphylococcus aureus: epidemiology, underlying mechanisms and associated risks. Clin Microbiol Rev. 1997 July;10(3):505-20. PMID: 9227864

4. Dan M, Moses Y, Poch F, Asherov J, Gutman R. Carriage of methicillinresistant Staphylococcus aureus by non-hospitalized subjects in Israel. Infection. 1992 Nov;20(6):332-35. doi: http://dx.doi.org/10.1007/ bf01710678 PMID: 1293052

5. Duncan IB, Collins AM, Neelin EM, Roy TE. Nasal carriage of Staphylococcus pyogenes by student nurses. Can Med Assoc J. 1957 Dec 1;77(11):1001-9. PMID: 13489588

6. McAnally TP, Lewis MR, Brown DR. Effect of rifampin and bacitracin on nasal carriers of Staphylococcus aureus. Antimicrob Agents Chemother. 1984 April; 25(4):22-6. PMID: 6732212

7. Paul MO, Lamikanra A, Aderibigbe DA. Nasal carriers of coagulase-positive staphylococci in a Nigerian hospital community. Trans R Soc Trop Med Hyg. 1982 Jan;76(3):319-23. doi: http://dx.doi.org/10.1016/0035-9203(82)901808 PMID: 7112654

8. Kumar P, Shukla I, Varshney S. Nasal screening of healthcare workers for nasal carriage of coagulase positive MRSA and prevalence of nasal colonization with Staphylococcus aureus. Biology and Medicine. 2011;3(2) Special Issue: 182-6.

9. Clinical and Laboratory Standards Institute. Performance standards for antimicrobial susceptibility testing. Eighteenth informational supplement. CLSI document M100-S22. Wayne, PA: Clinical and Laboratory Standards Institute; 2012

10. Chambers HF, Sachdeva M. Binding of beta-lactam antibiotics to penicillinbinding proteins in methicillin-resistant Staphylococcus aureus. J Infect Dis. 1990 Jun;161(6):1170-6.

11. Clinical and Laboratory Standards Institute: Performance Standards for Antimicrobial Susceptibility Testing. Fifteenth Informational Supplement January 2005. Approved Standard, M100-S15, Vol. 25, No. 1. Wayne, PA.

12. Kloos WE, Bannerman TL. Staphylococcus and Micrococcus. In: Murry PR, Baron EJ, Pfaller M, Tenover AFC, Yolken RK, editors. Manual of clinical microbiology, 7th ed. Washington, DC: American Society for Microbiology; 1999. pp. 264-82.

13. Monsen T, Rönnmark M, Olofsson C, Wiström, J. An inexpensive and reliable method for routine identification of staphylococcal species. Eur J Clin Microbiol Infect Dis. 1998 May;17(5):327-35. doi: http://dx.doi.org/10.1007/bf01709455 PMID: 9721961

14. Jain A, Agarwal A, Verma RK. Cefoxitin disc diffusion test for detection of meticillin-resistant staphylococci. J Med Microbiol. 2008 Aug;57 (Pt 8):957-61. doi: http://dx.doi.org/10.1099/jmm.0.47152-0 PMID: 18628495

15. Talan DA, Staatz D, Staatz A, Overturf GD. Frequency of Staphylococcus intermedius as human nasopharyngeal flora. J Clin Microbiol. 1989 Oct; 27(10):2393 PMID: 2584388 
16. Clinical Laboratory Standards Institute. Performance standard for antimicrobial susceptibility testing: seventeenth informational supplement M100-S17. Clinical Laboratory Standards Institute, Wayne, PA, USA; 2007.

17. Boelaert JR, Van Landuyt HW, De Baere YA, Deruyter MM, Daneels RF, Schurgers ML, et al. Staphylococcus aureus infections in hemodialysis patients: pathophysiology and use of nasal mupirocin for prevention. J Chemother. 1995 Jul;7 Suppl 3:49-53. PMID: 8609538

18. Koziol-Montewka M, Chudnicka A, Ksiazek A, Majdan M. Rate of Staphylococcus aureus nasal carriage in immunocompromised patients receiving hemodialysis treatment. Int J Antimicrob Agents. 2001 Aug;18(2):193-6. doi: http://dx.doi. org/10.1016/s0924-8579(01)00350-8 PMID: 11516945

19. Moellering RC Jr. Problems with antimicrobial resistance in gram-positive cocci. Clin Infect Dis. 1998 May:26(5):1177-8. PMID: 9597248

20. Uemura E, Kakinohana S, Higa N, Toma C, Nakasone N. Comparative characterization of Staphylococcus aureus isolates from throats and nose of healthy volunteers. Jpn J Infect Dis. 2004 Feb;57(1):21-4. PMID: 14985632

21. Soysal A, Sahin H, Yagci A, Barlan I, Bakir M. The low rate of methicillinresistant Staphylococcus aureus in Turkish children. Jpn J Infect Dis. 2006 Jun;59(3):195-6. PMID: 16785704

22. Onanuga A, Onaolapo J.A. Antimicrobial susceptibility of community associated Staphylococcus aureus isolates from healthy women in Zaria, Nigeria. Trop J Pharmaceutical Res. 2008 Apr 3;7(1):929-39. doi: http:// dx.doi.org/10.4314/tjpr.v7i1.14679

23. Pant J, Rai SK. Occurrence of Staphyloccous aureus in hospital environment and staffs in teaching hospital in Katmandu, Nepal. J Nepal Assoc. Medi Lab Sci. 2007;8:72-3.

24. Chigbu CO, Ezeronye OU. Antibiotic resistant Staphylococcus aureus in Abia state of Nigeria. Afr J Biotech. 2003 Oct 31;2(10):374-8. doi: http://dx.doi. org/10.5897/ajb2003.000-1077
25. Chatterjee SS, Ray P, Aggarwal A, Das A, Sharma M. A community-based study on nasal carriage of Staphylococcus aureus. Indian J Med Res. 2009 Dec;130(6):742-8. PMID: 20090137

26. Onanuga A, Temedie TC. Nasal carriage of multi-drug resistant Staphylococcus aureus in healthy inhabitants of Amassoma in Niger delta region of Nigeria. Afr Health Sci. 2011 Jun;11(2):176-81. PMID: 21857847

27. Adesida SA, Abioye OA, Bamiro BS, Brai BIC, Smith SI, Amisu KO, et al. Associated risk factors and pulsed field gel electrophoresis of nasal isolates of Staphylococcus aureus from medical students in a tertiary hospital in Lagos, Nigeria. Braz J Infect Dis. 2007 Feb;11(1):63-9. doi: http://dx.doi. org/10.1590/s1413-86702007000100016 PMID: 17625730

28. Ali M. Al-Dahbi, Harith J. Al-Mathkhury. Distribution of methicillin resistant Staphylococcus aureus in Iraqi patients and healthcare workers. Iraqi I Sci. 2013;54(2):293-300

29. Al-Geobory HA. Comparative study between methicillin resistant Staphylococcus aureus (MRSA) and methicillin sensitive Staphylococcus aureus (MSSA), and detect the antimicrobial effects of some plant extracts on them [MSc thesis]. Baghdad, Iraq: College of Science, Baghdad University; 2011.

30. Fey PD, Saïd-Salim B, Rupp ME, Hinrichs SH, Boxrud DJ, Davis CC, et al. Comparative molecular analysis of community or hospital-acquired methicillin-resistant Staphylococcus aureus. Antimicrob Agents Chemother. 2003 Jan;47(1):196-203.

31. Jain A, Agarwal A, Verma RK. Cefoxitin disc diffusion test for detection of meticillin-resistant staphylococci. J Med Microbiol. 2008 Aug;57(pt 8): 957-61. doi: http://dx.doi.org/10.1099/jmm.0.47152-0 PMID: 18628495 